\title{
THE MINI-DRIVER MODEL OF POLYGENIC CANCER EVOLUTION
}

Francesc Castro-Giner', Peter Ratcliffe2* and lan Tomlinson*1,3

IMolecular and Population Genetics Laboratory and ${ }^{3}$ NIHR Comprehensive Biomedical Research Centre, and ${ }^{2 H e n r y}$ Wellcome Building for Molecular Physiology, Nuffield Dept. of Clinical Medicine, University of Oxford, Roosevelt Drive, Oxford OX3 7BN, UK

*to whom correspondence should be addressed: pir@well.ox.ac.uk (PR) and iant@well.ox.ac.uk (IT)

\begin{abstract}
Much of cancer genetics has focussed on the identification of the most important somatic mutations ("major drivers") that cause tumour growth. However, many mutations found in cancer might not be major drivers or passenger mutations, but instead might have relatively weak tumour-promoting effects. Our aim herein is to highlight the existence of these mutations (termed 'mini-drivers' herein), as multiple mini-driver mutations might substitute for a major-driver change, for example in the presence of genomic instability or a high mutagen exposure. The mini-driver model has clinical implications, such as the likely limited effect of therapeutically targeting such genes. However, its main importance lies in helping to provide a complete understanding of tumorigenesis, especially since we anticipate that an increasing number of mini-driver mutations will be found by cancer genome sequencing.
\end{abstract}

\section{Introduction}

One of several striking features of tumorigenesis revealed by the sequencing of thousands of cancer exomes and genomes is that, compared with expectations, few new driver genes have been found to be mutated at a high frequency ${ }^{1-3}$. The long tail of cancer genes mutated at a low frequency might mask a large number of genes mutated at even lower frequencies, leading to proposals to sequence many thousands of cancers to generate a complete catalogue of driver mutations ${ }^{4,5}$. In part, these 
findings reflect the fact that the tumour environment is much more diverse than previously thought. Thus, any individual mutant gene may provide a large selective advantage in only a small proportion of tumours ${ }^{6}$, depending on factors such as different cells of cancer origin within one tissue, the existing complement of mutations and epigenetic changes, micro-environmental stress 7,8 and tumour-extrinsic factors such as hormones or diet 9,10 . However, the findings also raise an alternative possibility, namely that the somatic evolution of a cancer at times follows a gradualist or polygenic model: hence, rather than evolving stepwise owing to major-driver mutations of relatively large effects, the tumour steadily accumulates mutations, each of which provides a relatively modest selective advantage 4,11 . To put this another way, whilst we are used to thinking of cells having a few Achilles heels that cause tumorigenesis - namely the major-driver mutations in genes such as adenomatous polyposis coli (APC), von Hippel-Lindau (VHL), KRAS, TP53 and E-cadherin (CDHI) perhaps cancer evolution is more like that of whole organisms, in that many mutations are selected for small effects on evolutionary fitness, and are less likely to spread to fixation owing to the influences of genetic drift and changing environments. We refer to these mutations as "mini-drivers". Scenarios in which mini-driver mutations might act include the following: mutations in major-driver genes that have weaker or partial effects on function; optimising an existing functional defect by finetuning or amplifying the effects of mutations already present in the cancer cell; selectively removing "functional baggage", such as tumour-suppressive side-effects of major-driver mutations, or redundant or unnecessary cellular functions; and providing a competitive advantage within the cancer cell population, but not to the cancer as a whole. Whilst many of the different characteristics of "mini-driver" mutations have been proposed previously, our aim in this article is to develop and synthetise these suggestions in a single model.

\section{What is the mini-driver model?}

Let us assume that cancer-causing mutations — including somatically heritable epimutations provide the cell within the tumour with a replicative advantage compared with cells in normal tissue. Those mutations may also provide an advantage compared with other cells in the same tumour. Mutations that reliably and consistently provide large advantages will tend to be found frequently in that cancer type ${ }^{6}$. We refer to these as major-driver mutations, in contrast to the less frequent minidriver mutations, which are the focus of this article. In reality, there is likely to be a continuum of effects from driver mutations and we recognize that the dichotomous classification is one of convenience. However, we shall contrast major-driver and mini-drivers below, in order to highlight the roles and features of mini-drivers that, we believe, are likely to become increasingly apparent as 
cancer genome data accumulate. This dichotomy has proved useful in considering the frequencies and effect sizes of rare and common germline genetic variants in other contexts 12-14.

In the mini-driver mutation model, these mutations provide a small selective advantage to the cancer cell,, and hence tend to increase in frequency as tumorigenesis proceeds. However, they are nonessential for cancer growth, such that restoration of a specific mutant allele to wild type will not kill or "normalise" the tumour, or substantially reduce growth. The predicted features of mini-driver mutations (or indeed epimutations) include the following (Box I): firstly, they would be present in a small proportion of tumours; secondly, they would be present in sub-clones (because their weak selective advantage makes a selective sweep less likely); thirdly, they would show parallel evolution between cancer sub-clones and among cancers of the same type, and lastly, they would target functional motifs involved in processes such as regulation of gene expression, RNA stability, transcript switching, DNA methylation and other non-coding genomic features.

Clearly, not all somatic mutations that have these features will be mini-drivers. For example, certain major-drivers may occur rarely because their selective advantage is strongly context dependent, and conversely, most somatic mutations will be passengers (that is, with no effect on the tumour cell's fitness). However, evidence shows that somatic mutations outside coding DNA can act as driver mutations $15-20$, and a wealth of data from inherited cancers and model systems shows that changes to non-coding DNA can have small, but important, effects on disease phenotypes. It must therefore be kept in mind that some of the apparent somatic passenger mutations in promoters, enhancers, repressors, insulators, microRNAs, IncRNAs, untranslated regions, et cetera in cancers might be mini-driver mutations. Even apparently subtle changes in non-coding regions such as base substitutions might affect binding of important transcription factors, as has been shown by studies of cancer predisposition ${ }^{21}$.

What could mini-driver mutations do?

The range of mini-driver functions is potentially very broad, enabling or enhancing most of the proposed hallmarks of cancer 22 and several other functions. Here, we provide some hypothetical examples of how mini-drivers could act, and illustrate these with some real mutations that have not yet been proven to be mini-drivers, but for which a mini-driver role is plausible. In some cases, mutations might fulfil more than one of the roles below. 
The most straightforward type of mini-driver mutation to envisage is one that provides a functional derangement similar to - or overlapping with - that of a major driver, but in an attenuated form (Type I, Figure I, Table I). The mini-driver mutations might take the form of different mutant alleles in the same gene or mutations in a different gene in the same pathway as the major driver. In the KRAS oncogene, for example, most somatic mutations in cancer are found at codons 12 and 13 , with a few at codon 61 (http://cancer.sanger.ac.uk/cosmic/gene/analysis?ln=KRAS\#histo). However, it has increasingly become apparent that atypical mutations at codons 146 and I I7 occur in a few cancers despite those changes probably being less effective in activating the RAS/RAF/MEK/ERK pathway ${ }^{23}$. These atypical KRAS mutations are usually clonal (E. Domingo and I. Tomlinson, unpublished observations) and hence have not been driven out of the cancer cell population by any fitter mutations that might arise in codon 12 and 13 . The reasons for this remain unclear, but may include the following: additional mini-driver mutations may compensate for the deficiencies of the initial atypical (mini-driver) variant; the atypical mutant has a "head start" if it occurs before the typical mutation, causing clonal and physical expansion and acquiring additional driver changes, which a clone carrying the typical mutation cannot overhaul or displace; and, finally, typical and atypical mutations in the same cell may together over-activate the pathway beyond an optimum and hence reduce fitness.

Another type of mini-driver mutation, Type II (Figure I, Table I), might optimise an existing functional defect by fine-tuning or amplifying the effects of mutations already present in the cancer cell so that fitness is increased. For instance, such mini-driver modifier mutations might keep signalling pathway levels at an optimum in a changing selective landscape, or modulate the level of genomic instability upwards or downwards as the cancer population expands, or negate the effects of deleterious mutations that have increased in frequency by hitchhiking or genetic drift through the population. As with major drivers, cell autonomous advantage may arise from the context-specific balance of multiple pro- and anti-tumorigenic effects, but is more finely balanced so that contextual specificity is even more pronounced. A potential example here comes from colorectal cancer (CRC), in which the Wnt pathway is almost universally activated by APC tumour suppressor mutations 24,25 . There appears to be an optimum level of Wnt signalling for tumorigenesis, in which the pathway is activated to an intermediate level, initially by specific combinations of APC mutations that differentially affect degradation of the Wnt effector $\beta$-catenin $26-28$ Some cancers acquire mutations additional to the "two hits" at APC — including copy number gains and deletions involving APC in polyploid cells ${ }^{29}$ and inactivating mutations in the Wnt target gene SOX 9 - that are expected to reduce some of the effects of Wnt activation ${ }^{25} .30$ 
A related, and particularly intriguing, role for mini-driver mutations (Type III, Figure I, Table I) is to selectively remove tumour growth suppressing side-effects of major driver mutations. The rationale for this suggestion is that major driver mutations almost inevitably have a wide range of effects, not all of which promote tumorigenesis, even if the net effect is strongly positive. In a related scenario, a major driver function might have once been advantageous, but have become disadvantageous. In both of these cases, there is thus selection pressure to remove the effects of the "functional baggage". One example is the acquisition of polyploidy by cancers, which is postulated to be selected as an initial genome doubling, usually followed by loss of material such that cells end up in a neartriploid state ${ }^{31}$. Another possible example comes from mutations in the tumour suppressor VHL, which are almost ubiquitous in clear cell renal cell carcinoma (CCRCC) and have several effects, one of which is constitutive activation of the hypoxia signalling pathway through stabilisation of hypoxia inducible factor (HIF) $\alpha$ sub-units (HIF-I $\alpha$ and HIF-2 $\alpha$ ) ${ }^{32}$. Whilst the HIF-1 $\alpha$ isoform is generally thought to promote tumorigenesis, VHL-defective CCRCC shows an unusual bias towards increased HIF- $2 \alpha$ (not HIF-1 $\alpha$ ) expression. Indeed some renal cancers acquire inactivating HIF1A mutations ${ }^{33}$. This suggests that very strong constitutive activation of HIF pathways following VHL-inactivation induces tumour-restricting side-effects arising at least in part from the HIF-1 $\alpha$ component of the transcriptional cascade. This is consistent with selection for subsequent removal of that specific consequence of $\mathrm{VHL}$ mutation.

Another class of mini-driver mutation (Type IV, Figure I, Table I) could also more generally remove redundant or unnecessary functions, such as activities of the cancer progenitor cell that no longer serve any "useful" purpose for the cancer or even passenger mutations 34 that were once selectively neutral but became deleterious. There are case reports in the literature of apparent loss of driver mutations as cancers progress, as exemplified by a pathogenic IDHI mutation in a post-treatment glioblastoma ${ }^{35}$. Furthermore, the high frequency of inactivating somatic mutations in some genes with a role in differentiated cells and no plausible role in carcinogenesis ${ }^{2}$, may not always reflect factors like the large size of some of these genes and their tolerance of mutations, but instead a nontrivial benefit to the cell as it no longer has to make the proteins encoded by these genes.

As a final example, we include in a Type $\mathrm{V}$ (Figure I, Table I) mini-driver mutation group those mutations that provide a competitive advantage within the set of cells that comprise the cancer, but provide a minimal advantage to the cancer as a whole. There are currently few, if any, proven examples of this, but circumstantial evidence and experiments in model systems increasingly favour the importance of competition between tumour cells. Mini-driver mutations of this type might include "freeloaders" - for example, cells that utilise but do not produce paracrine growth factors and cytokines - or one cell population that can directly harm another ${ }^{36-41}$. A classic example of 
antagonism among tumour cells was described in syngeneic mice injected with mixtures of two mammary tumour cell lines (168 and 4T07); 168 cell growth was suppressed by growth inhibitory factors generated by 4T07 cells ${ }^{42}$. In other cases, though, mini-driver mutations might act to promote co-operation among tumour cells ${ }^{37,40,43-46}$, which could be essential for tumour persistence and might facilitate processes such as invasion and metastasis $47-49$.

What mini-driver mutations are not

For clarity, the mini-driver category of mutations is not defined by the proportion of cancers that harbour mutations, or by the presence of mutation in the major clone or a sub-clone. Major drivers might be mutated in a small proportion of cancers, but still confer a large selective advantage, for instance if they are poorly mutable, or only selected in certain environments, genetic backgrounds or cells of origin. Similarly, sub-clonal drivers may confer large selective advantages, but they are selected in certain environments, genetic backgrounds or cells of origin, or at a particular stage of tumorigenesis. It is not uncommon for a specific mutation to be a well-established, frequent major driver in one cancer, but to occur very uncommonly in other cancer types. An example is missense mutations at codon I 32 of isocitrate dehydrogenase I (IDHI), which are found commonly in some malignancies, principally glioblastoma ${ }^{50}$ and acute myeloid leukaemia ${ }^{51}$, but are also present in a very few CRCs, breast cancers, bladder cancers and lung cancers ${ }^{6}$. The latter IDHI mutations might be major or mini-drivers, depending on the magnitude of their functional effects.

\section{Identifying mini-driver mutations}

Since individual mini-driver mutations may well be uncommon across cancers, context dependent, sub-clonal, outside coding regions, and over-represented in cancers with a high background mutation rate, demonstrating their over-representation statistically in the cancer genome is likely to be challenging, despite some promising starts (for example, ${ }^{52}$ ). Nevertheless, the provision of such statistical evidence remains a highly desirable scientific goal, and is an important justification, additional to those already put forward, for sequencing of cancer genomes rather than exomes and for studying bigger sample sizes extending to thousands of tumours. In addition, information to support statistically over-represented mutations is likely to be especially important for mini-drivers, and multiple different types of evidence may be marshalled. More efficient exploration of genomic data, such as methods exploring the co-occurrence of alterations across pathways and identification of altered networks might help to identify driver events occurring at a very low frequency $2,53-55$. 
Those methods reduce the number of hypotheses tested and thus, increase the statistical power to identify rare mutated events. A pan-cancer network analysis was able to identify networks containing rarely mutated genes that were not previously identified by single-gene test methods 3, 56-58. Type I, II and III mini-drivers are related to major drivers and hence have a low prior threshold for investigation. For example, our own analysis of CRC data from The Cancer Genome Atlas (TCGA) shows that cancers wildtype for major drivers in the PI3K/Akt pathway (KRAS, BRAF, PIK3CA, PIK3RI, PTEN, and NRAS) tend to have multiple mutations in other pathway members such as PIK3CG, OSMR, HGF, LAMBI and JAK3 ( $\mathrm{P}=0.03$, IntOGen pathway analysis ${ }^{59}$ ). Type IV mini-driver mutations (where redundant functions are lost) probably have limited clinical relevance and will be especially hard to find. However, Type $V$ mini-drivers (effects within the cancer cell population) may be more tractable to identification based on prior knowledge of gene function.

In addition to statistical and bioinformatic approaches, sensitive functional assays will be required. Technologies such as CRISPR-Cas9 (clustered regularly interspaced short palindromic repeatsCRISPR-associated 9) allow the introduction of multiple mutations into specific genetic and environmental cellular contexts, and more sophisticated assays, including those that analyse competition between cells with different genotypes, have great promise for identifying subtle differences in cellular properties. We contend that greater scientific efforts should go into measuring the functional effects of different mutations, including context-specific mutation fitness.

\section{The somatic polygenic model of tumorigenesis}

Whilst we envisage that mini-driver mutations exist in many cancers, it is, at the extreme, consistent with a model in which a large number of mutations, each of them associated with a weak fitness advantage, would drive tumorigenesis 4, 11,52,60. By analogy with cancer predisposition, this scenario would be akin to a Mendelian cancer phenotype resulting from the inheritance of multiple risk alleles of modest effect rather than a single high-risk mutation 61 - in other words, a polygenic model. More generally, we propose that many cancers follow a polygenic model at least in part. Elements of this model have been proposed independently. For example, Davoli et al ${ }^{52}$ recently suggested that single mutations, such as large copy number changes that simultaneously affect multiple weak driver genes, could cumulatively have an effect equal to a single potent driver mutation.

We hypothesise that certain factors would favour a somatic polygenic model of tumorigenesis based on multiple mini-driver mutations: first, a high somatic mutation rate, whether from genomic instability or the environment, especially one favouring nucleotide substitutions different from those 
typically observed in the common driver mutations for a given cancer type ${ }^{62}$; second, a heterogeneous and/or changing environment, broadly defined to include intra- and extra-cellular factors 7,8,63; and third, a large pool of mini-driver genes that could provide a selective advantage in that cell or tissue type, in effect raising the mutation rate. These conditions would promote a high frequency of sub-optimal, but selected, mutations, the relatively easy acquisition of compensating mutations, and rapid adaptation through fine-tuning or amplification of sub-optimal mutations. However, we suspect that a large stochastic element, such as genetic drift or bottlenecks, might be required to prevent major driver mutations from spreading and taking over the tumour population, especially relatively early in tumorigenesis, when the tumour is small and growing slowly $64-67$, and thus newly-arisen mutant tumour clones are at great risk of dying out by chance. This scenario favours the survival of individual mutations with relatively big effects on fitness. Later in tumorigenesis, the tumour is growing faster ${ }^{64-66,68}$, micro-environmental stresses are likely to be more dynamic ${ }^{69}$, and the mutant has a greater chance of surviving, even if its selective advantage is very modest or close to zero. Tumour size and, sometimes, genetic instability mean that later in tumorigenesis, many "mini-driver" mutations with small effects might arise and persist in the cancer cell population, and may spread through it to fixation or high frequency in part or all of the tumour.

One potential situation in which a mini-driver model might apply is in so-called "ultramutator" colorectal and endometrial cancers that have defective DNA polymerase proof-reading, a shifted mutation spectrum and a burden of about $1,000,000$ base substitution mutations at levels detectable by genome sequencing ${ }^{25}$. Many ultramutator cancers have conventional major driver mutations in genes such as APC, TP53 and F-box and WD repeat domain containing 7 (FBXW7) ${ }^{62}$. However, there is a tendency for these cancers to acquire atypical mutations, such as a high frequency of mutations at unusual sites in the known major driver genes (e.g. codon I46 and II 7 of KRAS and codon 88 of PIK3CA), and these are potential mini-driver changes. In addition, some ultramutator cancers have apparently pathogenic mutations that are more typical of other cancer types (e.g. ultramutator endometrial cancers often activate Wnt not through the usual means of CTNNBI mutation but by APC inactivation, which is more typical of colorectal cancers ${ }^{62}$ ). A further piece of evidence in favour of a polygenic model for ultramutator CRC and endometrial cancers is that they have an unusually high proportion of non-synonymous mutations compared with synonymous changes (NS:S ratio, analysis in Figure 2; data from ${ }^{25,70}$ ). Although this finding requires cautious interpretation because the specific mutational biases in ultramutator cancers are not well characterised, it suggests that these cancers in fact have many mutations of potential functional effect, consistent with a mini-driver model, contrary to expectations that a high mutational load would burden these tumours and hence cause a low NS:S ratio. 
Mutagenesis screens in mice also provide some support for the polygenic model. Such screens to identify genes that promote or suppress tumorigenesis have been performed in animal models for many years, historically using mutagens and/or carcinogens. More recently, transposon-mediated mutagenesis screens using Sleeping Beauty or Piggy-BAC elements have identified hundreds of loci that promote tumour growth when disrupted in a near-random fashion ${ }^{71}$. This number is far in excess of the number of high-confidence driver genes for any human cancer type, and these mice frequently carry transposon-mediated disruptions of many genes. Although the high effective mutation rates of such screens may favour combinations of relatively weak mutations compared with the situation in spontaneous tumorigenesis, the mutagenesis screen data are consistent with the principle that multiple mini-driver mutations can substitute functionally for major drivers.

\section{Clinical implications of the mini-driver model}

One of the main justifications for large-scale cancer genome sequencing is that it will yield many new, useful anti-cancer targets. There is still great promise in this regard, largely through driver mutations that are uncommon but that may have major effects - examples include the set of PBAF (polybromo-associated BRGI- or HRBM-associated factor) chromatin remodelling complex genes, such as ARIDIA, ARIDIB, ARID2, SMARCA4 and PBRMI, that are mutated in several different cancer types 72 . However, should many of the "new" cancer genes turn out to be mini-drivers, the therapeutic potential for mutation-targeted treatments is evidently more limited. This is especially true if a cancer has arisen primarily through a polygenic model, in which case targeting one or a few mini-drivers is unlikely to be very successful, unless a shared carcinogenic pathway can be targeted. Conversely, such cancers might intrinsically have a good prognosis owing to their mutational load and it is possible to envisage successful, new treatment strategies with combinations of agents that have relatively low toxicity.

\section{Conclusions}

There are good reasons to expect that many mutations found in cancer are not major drivers of tumour growth or irrelevant passenger mutations, but instead are changes that are selected, yet have relatively weak effects. We have provided some scenarios in which these mini-driver mutations might act, and a few examples of cancer mutations that might turn out to be mini-drivers. In many cases, we suspect that the current mini-driver examples modulate the effects of major drivers. However, we also hypothesise that in some cancers, multiple mini-driver mutations may contribute 
to a polygenic model of tumorigenesis, in which many mini-driver mutations, rather than a few major drivers, promote tumour growth. Underlying severe genomic instability is one situation in which we would expect a polygenic form of tumorigenesis to be relatively likely.

In our collective opinion, increased research into mini-driver mutations is highly desirable and may provide important insights into the fundamentals of tumorigenesis. However, obtaining evidence for the mini-driver model may not be straightforward. It will probably require the initial identification of potential mini-drivers based on modestly elevated mutation frequency relative to background and/or a functional relationship to known driver genes. Subsequent in vitro assays to test a mini-driver mutation's effects are necessary, but may be challenging given the small functional changes involved and the possible context-dependency of the mini-driver change. It is possible that genetically engineered animal models of cancer provide the best chance of a suitable quantitative read-out here, as long as they accurately reflect sporadic tumorigenesis which is a relatively rare event that affects a small number of specific cells.

The mini-driver model does have clinical implications, but its main importance lies in helping to provide a complete understanding of tumorigenesis. The existence of mini-drivers is predicted by classical evolutionary theory, and there are several parallels here with cancer predisposition, in which there exist a variety of genetic variants with different frequencies and sizes of effect ${ }^{14}$. Our purpose has been to highlight the likely existence of mini-driver mutations and to propose how and why they might be selected. We anticipate that an increasing number of mini-drivers will be found and that this process will provide important insights into tumorigenesis.

\section{Acknowledgements}

We are very grateful to Trevor Graham for comments and criticism. Core funding to the Wellcome Trust Centre for Human Genetics was provided by the Wellcome Trust (090532/Z/09/Z).

\section{Competing Interests}

None of the authors has a competing interest to declare. 


\section{Author Biographies}

Francesc Castro-Giner joined the Molecular and Population Genetics laboratory at the Wellcome Trust Centre for Human Genetics, University of Oxford, UK, in January 2013 as postdoctoral scientist. He earned his Ph.D. in 2009 from the Universitat Pompeu Fabra, Barcelona, Spain, and trained as a postdoctoral fellow at the Centre Nacional D'Anàlisi Genòmica, Barcelona, Spain. His interests are in cancer genomics, cancer evolution, complex diseases genetics and computational biology.

Peter Ratcliffe is Nuffield Professor of Clinical Medicine at the University of Oxford and a member of the Ludwig Institute of Cancer Research. His laboratory played a central role in the elucidation of oxygen sensing pathways that regulate the cell's transcriptional program in hypoxia. His current research interests include not only the biology of hypoxia, but also experimental and theoretical aspects of carcinogenesis, especially the implications of oncogenic 'switching' of massive physiological pathways during tumour development.

lan Tomlinson is Professor of Molecular and Population Genetics at the University of Oxford. His interests include identification of high- and low-penetrance tumour predisposition genes and functional cancer genetics. He has a longstanding interest in cancer evolution, including simple mathematical and descriptive models, and experimental analysis of tumour genomes. 


\section{Display Items}

Box I. Summary of the mini-driver model

- Mini-driver mutations positively influence tumour cell replication, but are not critical for it

- Multiple mini-drivers may sometimes substitute for one or a few major drivers during tumorigenesis (polygenic model)

- Mini-drivers may be more influenced by genetic drift than major drivers

- Mini-drivers may be over-represented outside coding regions

- Individual mini-driver mutations are likely to be uncommon in cancers of any one type, but many mini-drivers may be present in a cancer

- Mini-drivers will tend to be present in sub-clones, perhaps showing parallel or convergent evolution between cancer sub-clones

Box 2. Possible sources of evidence for the mini-driver model

- Genetic: e.g. substitutes for major drivers or modulators of major drivers, multiple mutations in same pathway, same pathways involved when major drivers excluded

- Evolutionary: associated with mutator phenotypes, higher than expected frequency of nonsynonymous substitutions

- Functional: weaker effects of variants on key cancer-promoting functions 


\section{Figure legends}

Figure I. Schemas of some mini-driver scenarios.

In Type I mini-driver mutations (left, upper), an attenuated version of a major-driver is combined with other mini-drivers to provide a selective advantage equivalent to a single major-driver mutation. In Types II, III and IV (right, upper), the mini-driver mutation in a sub-clone optimises the effects of a major-driver (Type II), selectively removes tumour growth suppressing side-effects of a major-driver mutations (Type III), or removes redundant functions (Type IV), leading faster growth of that subclone. In Type V, mini-driver mutations can lead to cell death of other sub-clones through competition (left, lower) or co-operatively promote the growth of other cells (right, lower).

Figure 2. Ratio of non-synonymous:synonymous mutations and mutational load in colorectal cancers according to their mutation burden

Data are from colorectal cancer exome sequencing data from The Cancer Genome Atlas (TCGA) ${ }^{25}$ and comprise somatic single nucleotide variants (SNVs) (Cancer Genomics Hub (CGHub; https://cghub.ucsc.edu/) repository, accessed on $5^{\text {th }}$ December 20I4). Cancers were classified as ultramutator with polymerase proofreading mutations (in polymerase epsilon (POLE)), microsatelliteinstability positive (MSI+), wildtype for all major-driver mutations (WT) and the remaining group of about $80 \%$ of all cancers (Other). The box-and-whiskers plots show NS:S ratio (y-axis). The ultramutator tumours have a significantly higher NS:S ratio than the three other groups $(P=0.001$ versus $\mathrm{MSI}+, \mathrm{P}=0.003$ versus $\mathrm{WT}, \mathrm{P}=0.008$ versus Other, $\mathrm{t}$ test)., $\mathrm{t}$ test). The $\mathrm{MSI}+$ group do not have a significantly higher NS:S ratio compared with all other groups combibed, but do additionally have a much higher indel mutation burden. The data for endometrial cancer (not shown) are very similar. 
Table I. Summary table of proposed exemplar mini-drivers

\begin{tabular}{|c|c|c|c|}
\hline Type & Definition & Effect & Possible examples from sporadic human cancers \\
\hline 1 & $\begin{array}{l}\text { Attenuated form of major- } \\
\text { driver mutation }\end{array}$ & $\begin{array}{l}\text { Mutations in the same gene or pathway } \\
\text { as the major driver }\end{array}$ & KRAS mutations at codons 146 and II 23 \\
\hline \multirow[t]{4}{*}{ II } & \multirow[t]{4}{*}{$\begin{array}{l}\text { Optimising major-driver } \\
\text { mutations }\end{array}$} & $\begin{array}{l}\text { Keep signalling pathway levels at an } \\
\text { optimum }\end{array}$ & $\begin{array}{l}\text { Reduction of the effects of Wnt activation, through CNA in } \\
A P C{ }^{29} \text { and inactivating mutations in SOX } 925\end{array}$ \\
\hline & & $\begin{array}{l}\text { Modulate the level of genomic } \\
\text { instability }\end{array}$ & None known at this time \\
\hline & & $\begin{array}{l}\text { Reverse the effects of deleterious } \\
\text { hitchhikers }\end{array}$ & None known at this time \\
\hline & & Amplifying germline variation & $\begin{array}{l}\text { Targeting a polymorphism that affected an enhancer that } \\
\text { drives an oncogene, such as allelic imbalance at rs6983267 } \\
\text { contributing to CRC somatic evolution } 30\end{array}$ \\
\hline \multirow[t]{2}{*}{ III } & \multirow[t]{2}{*}{$\begin{array}{l}\text { Selectively remove } \\
\text { disadvantageous major-driver } \\
\text { functions }\end{array}$} & $\begin{array}{l}\text { Remove growth suppressing side } \\
\text { effects of driver mutations }\end{array}$ & $\begin{array}{l}\text { Renal cancers acquiring inactivating HIFIA mutations } \\
\text { consistent with selection for removal of that specific } \\
\text { consequence of } V H L \text { mutation } 33\end{array}$ \\
\hline & & $\begin{array}{l}\text { Remove major-driver function that has } \\
\text { become disadvantageous during } \\
\text { somatic evolution }\end{array}$ & None known at this time \\
\hline \multirow[t]{2}{*}{ IV } & \multirow[t]{2}{*}{$\begin{array}{l}\text { Remove redundant or } \\
\text { unnecessary functions }\end{array}$} & $\begin{array}{l}\text { Loss of driver mutations that are no } \\
\text { longer needed as cancers progress }\end{array}$ & $\begin{array}{l}\text { Loss of IDHI from glioblastoma (although not therapy- } \\
\text { naïve) }{ }^{35}\end{array}$ \\
\hline & & $\begin{array}{l}\text { High frequency of inactivating somatic } \\
\text { mutations in some genes with a role in } \\
\text { differentiated cells and no plausible role } \\
\text { in carcinogenesis }\end{array}$ & None known at this time \\
\hline \multirow[t]{2}{*}{ V } & \multirow{2}{*}{$\begin{array}{l}\text { Competition or co-operation } \\
\text { between tumour cells with } \\
\text { minor overall effect }\end{array}$} & $\begin{array}{l}\text { "Freeloading" or one cell population } \\
\text { directly harming another }\end{array}$ & None known at this time \\
\hline & & $\begin{array}{l}\text { Promote co-operation among tumour } \\
\text { cells }\end{array}$ & None known at this time \\
\hline
\end{tabular}

APC, adenomatous polyposis coli; CNA, copy number alteration; CRC, colorectal cancer; HIFIA, hypoxia-inducible factor I $\alpha$; IDHI, isocitrate dehydrogenase I; VHL, von Hippel-Lindau 


\section{References}

I. Garraway, L.A. \& Lander, E.S. Lessons from the cancer genome. Cell I53, I7-37 (2013).

2. Leiserson, M.D. et al. Pan-cancer network analysis identifies combinations of rare somatic mutations across pathways and protein complexes. Nat Genet 47, I06-I4 (20I5).

3. Vogelstein, B. et al. Cancer genome landscapes. Science 339, I546-58 (2013).

4. Stratton, M.R., Campbell, P.J. \& Futreal, P.A. The cancer genome. Nature 458, 7I 9-24 (2009).

5. Marx, V. Cancer genomes: discerning drivers from passengers. Nat Methods I I, 375-9 (2014).

6. Lawrence, M.S. et al. Discovery and saturation analysis of cancer genes across $2 \mathrm{I}$ tumour types. Nature 505, 495-50I (2014).

7. Greaves, M. \& Maley, C.C. Clonal evolution in cancer. Nature 48 I, 306-I3 (20I2).

8. Merlo, L.M., Pepper, J.W., Reid, B.J. \& Maley, C.C. Cancer as an evolutionary and ecological process. Nat Rev Cancer 6, 924-35 (2006).

9. Amos-Landgraf, J.M. et al. Sex disparity in colonic adenomagenesis involves promotion by male hormones, not protection by female hormones. Proc Natl Acad Sci U S A I I I, I65 I 4-9 (20|4).

10. Figueiredo, J.C. et al. Genome-wide diet-gene interaction analyses for risk of colorectal cancer. PLoS Genet I 0, el 004228 (20 I4).

II. Beerenwinkel, N. et al. Genetic progression and the waiting time to cancer. PLoS Comput Biol 3, e225 (2007).

12. Bansal, V., Libiger, O., Torkamani, A. \& Schork, N.J. Statistical analysis strategies for association studies involving rare variants. Nat Rev Genet I I, 773-85 (20 I0).

13. Bodmer, W. \& Bonilla, C. Common and rare variants in multifactorial susceptibility to common diseases. Nat Genet 40, 695-70I (2008).

14. Hindorff, L.A., Gillanders, E.M. \& Manolio, T.A. Genetic architecture of cancer and other complex diseases: lessons learned and future directions. Carcinogenesis 32, 945-54 (201 I).

15. Cheetham, S.W., Gruhl, F., Mattick, J.S. \& Dinger, M.E. Long noncoding RNAs and the genetics of cancer. Br J Cancer I 08, 24I9-25 (2013).

16. Fredriksson, N.J., Ny, L., Nilsson, J.A. \& Larsson, E. Systematic analysis of noncoding somatic mutations and gene expression alterations across 14 tumor types. Nat Genet 46, 1258-63 (2014).

17. Hayes, J., Peruzzi, P.P. \& Lawler, S. MicroRNAs in cancer: biomarkers, functions and therapy. Trends Mol Med 20, 460-9 (20I4).

18. Horn, S. et al. TERT promoter mutations in familial and sporadic melanoma. Science 339, 959-6I (2013).

19. Huang, F.W. et al. Highly recurrent TERT promoter mutations in human melanoma. Science 339, 957-9 (2013).

20. Weinhold, N., Jacobsen, A., Schultz, N., Sander, C. \& Lee, W. Genome-wide analysis of noncoding regulatory mutations in cancer. Nat Genet 46, II 60-5 (20I4).

21. Zeron-Medina, J. et al. A polymorphic p53 response element in KIT ligand influences cancer risk and has undergone natural selection. Cell I55, 4I0-22 (20I3).

22. Hanahan, D. \& Weinberg, R.A. Hallmarks of cancer: the next generation. Cell I44, 646-74 (20II).

23. Imamura, Y. et al. Analyses of clinicopathological, molecular, and prognostic associations of KRAS codon $6 \mathrm{I}$ and codon I46 mutations in colorectal cancer: cohort study and literature review. Mol Cancer I 3, I 35 (2014).

24. Sansom, O. Tissue-specific tumour suppression by APC. Adv Exp Med Biol 656, 107-I8 (2009).

25. TCGA. Comprehensive molecular characterization of human colon and rectal cancer. Nature 487, 330-7 (20I2).

26. Lamlum, $\mathrm{H}$. et al. The type of somatic mutation at APC in familial adenomatous polyposis is determined by the site of the germline mutation: a new facet to Knudson's 'two-hit' hypothesis. Nat Med 5, I07I-5 (1999). 
27. Albuquerque, $C$. et al. The 'just-right' signaling model: APC somatic mutations are selected based on a specific level of activation of the beta-catenin signaling cascade. Hum Mol Genet I I, I549-60 (2002).

28. Schneikert, J., Grohmann, A. \& Behrens, J. Truncated APC regulates the transcriptional activity of beta-catenin in a cell cycle dependent manner. Hum Mol Genet 16, 199-209 (2007).

29. Segditsas, S. et al. APC and the three-hit hypothesis. Oncogene 28, I46-55 (2009).

30. Tuupanen, S. et al. Allelic imbalance at rs6983267 suggests selection of the risk allele in somatic colorectal tumor evolution. Cancer Res 68, I4-7 (2008).

31. Dewhurst, S.M. et al. Tolerance of whole-genome doubling propagates chromosomal instability and accelerates cancer genome evolution. Cancer Discov 4, I75-85 (2014).

32. Pugh, C.W. \& Ratcliffe, P.J. The von Hippel-Lindau tumor suppressor, hypoxia-inducible factor-I (HIF-I) degradation, and cancer pathogenesis. Semin Cancer Biol I3, 83-9 (2003).

33. Shen, $\mathrm{C}$. et al. Genetic and functional studies implicate HIFIalpha as a I4q kidney cancer suppressor gene. Cancer Discov I, 222-35 (20II).

34. McFarland, C.D., Korolev, K.S., Kryukov, G.V., Sunyaev, S.R. \& Mirny, L.A. Impact of deleterious passenger mutations on cancer progression. Proc Natl Acad Sci U S A I I 0, 29I 0-5 (20I3).

35. Favero, F. et al. Glioblastoma adaptation traced through decline of an IDHI clonal driver and macroevolution of a double minute chromosome. Ann Oncol (20I5). In press.

36. Korolev, K.S., Xavier, J.B. \& Gore, J. Turning ecology and evolution against cancer. Nat Rev Cancer I4, 37I-80 (20I4).

37. Marusyk, A. et al. Non-cell-autonomous driving of tumour growth supports sub-clonal heterogeneity. Nature 5 I 4, 54-8 (20 I4).

38. Reilly, K.M. \& Van Dyke, T. It takes a (dysfunctional) village to raise a tumor. Cell I35, 40810 (2008).

39. Tabassum, D.P. \& Polyak, K. Tumorigenesis: it takes a village. Nat Rev Cancer (20I5).

40. Tomlinson, I.P. Game-theory models of interactions between tumour cells. Eur J Cancer 33, 1495-500 (1997).

4I. Tomlinson, I.P. \& Bodmer, W.F. Modelling the consequences of interactions between tumour cells. Br J Cancer 75, I57-60 (1997).

42. Miller, B.E., Miller, F.R., Wilburn, D. \& Heppner, G.H. Dominance of a tumor subpopulation line in mixed heterogeneous mouse mammary tumors. Cancer Res 48, 5747-53 (1988).

43. Axelrod, R., Axelrod, D.E. \& Pienta, K.J. Evolution of cooperation among tumor cells. Proc Natl Acad Sci U S A I 03, I3474-9 (2006).

44. Cleary, A.S., Leonard, T.L., Gestl, S.A. \& Gunther, E.J. Tumour cell heterogeneity maintained by cooperating subclones in Wnt-driven mammary cancers. Nature 508, II 3-7 (2014).

45. Inda, M.M. et al. Tumor heterogeneity is an active process maintained by a mutant EGFRinduced cytokine circuit in glioblastoma. Genes Dev 24, I73 I-45 (2010).

46. Turke, A.B. et al. Preexistence and clonal selection of MET amplification in EGFR mutant NSCLC. Cancer Cell I7, 77-88 (2010).

47. Aceto, N. et al. Circulating tumor cell clusters are oligoclonal precursors of breast cancer metastasis. Cell I58, III 0-22 (20I4).

48. Calbo, J. et al. A functional role for tumor cell heterogeneity in a mouse model of small cell lung cancer. Cancer Cell 19, 244-56 (201I).

49. Marusyk, A. \& Polyak, K. Tumor heterogeneity: causes and consequences. Biochim Biophys Acta I805, I05-17 (20I0).

50. Parsons, D.W. et al. An integrated genomic analysis of human glioblastoma multiforme. Science 32 I, I807-12 (2008).

5I. Mardis, E.R. et al. Recurring mutations found by sequencing an acute myeloid leukemia genome. N Engl J Med 36 I, I058-66 (2009).

52. Davoli, T. et al. Cumulative haploinsufficiency and triplosensitivity drive aneuploidy patterns and shape the cancer genome. Cell I 55, 948-62 (20I3).

53. Creixell, P. et al. Pathway and network analysis of cancer genomes. Nat Methods I 2, 6I5-2 I (20I5). 
54. Krogan, N.J., Lippman, S., Agard, D.A., Ashworth, A. \& Ideker, T. The Cancer Cell Map Initiative: Defining the Hallmark Networks of Cancer. Mol Cell 58, 690-698 (20I5).

55. Pe'er, D. \& Hacohen, N. Principles and strategies for developing network models in cancer. Cell I44, 864-73 (20II).

56. Gonzalez-Perez, A. \& Lopez-Bigas, N. Functional impact bias reveals cancer drivers. Nucleic Acids Res 40, el69 (20I2).

57. Lawrence, M.S. et al. Mutational heterogeneity in cancer and the search for new cancerassociated genes. Nature 499, 2I4-8 (20I3).

58. Tamborero, D., Lopez-Bigas, N. \& Gonzalez-Perez, A. Oncodrive-CIS: a method to reveal likely driver genes based on the impact of their copy number changes on expression. PLoS One 8, e55489 (2013).

59. Gundem, G. et al. IntOGen: integration and data mining of multidimensional oncogenomic data. Nat Methods 7, 92-3 (2010).

60. Salk, J.J., Fox, E.J. \& Loeb, L.A. Mutational heterogeneity in human cancers: origin and consequences. Annu Rev Pathol 5, 5I-75 (20I0).

6I. Balmain, A., Gray, J. \& Ponder, B. The genetics and genomics of cancer. Nat Genet 33 Suppl, 238-44 (2003).

62. Heitzer, E. \& Tomlinson, I. Replicative DNA polymerase mutations in cancer. Curr Opin Genet Dev 24, I07-I3 (2014).

63. Anderson, A.R., Weaver, A.M., Cummings, P.T. \& Quaranta, V. Tumor morphology and phenotypic evolution driven by selective pressure from the microenvironment. Cell I27, 905-I5 (2006).

64. Sidow, A. \& Spies, N. Concepts in solid tumor evolution. Trends Genet 3I, 208-I4 (20I5).

65. Reiter, J.G., Bozic, I., Allen, B., Chatterjee, K. \& Nowak, M.A. The effect of one additional driver mutation on tumor progression. Evol Appl 6, 34-45 (2013).

66. Bozic, l. et al. Accumulation of driver and passenger mutations during tumor progression. Proc Natl Acad Sci U S A I 07, I8545-50 (2010).

67. Crespi, B. The evolutionary biology of child health. Proc Biol Sci 278, I44I-9 (20II).

68. Peer, P.G., van Dijck, J.A., Hendriks, J.H., Holland, R. \& Verbeek, A.L. Age-dependent growth rate of primary breast cancer. Cancer 7 I, 3547-5I (1993).

69. Egeblad, M., Nakasone, E.S. \& Werb, Z. Tumors as organs: complex tissues that interface with the entire organism. Dev Cell I8, 884-90I (2010).

70. Kandoth, C. et al. Integrated genomic characterization of endometrial carcinoma. Nature 497, 67-73 (20I3).

7I. Abbott, K.L. et al. The Candidate Cancer Gene Database: a database of cancer driver genes from forward genetic screens in mice. Nucleic Acids Res 43, D844-8 (20I5).

72. Shain, A. \& Pollack, J. The spectrum of SWI/SNF mutations, ubiquitous in human cancers. PLOS ONE 8, e55II 9 (20I3). 